\title{
Atomic forces from electronic energies via the Hellmann-Feynman theorem, with application to semiconductor (110) surface relaxation
}

\author{
Otto F. Sankey \\ Department of Physics, Arizona State University, Tempe, Arizona 85287 \\ Roland E. Allen \\ Department of Physics, Texas A\&M University, College Station, Texas 77843-4242
}

(Received 8 July 1985)

\begin{abstract}
A method has been devised for computer simulations of covalently bonded systems, such as semiconductors. The method uses noncentral and nonlocal effective potentials generated from the electronic structure via the Hellmann-Feynman theorem. As an elementary example, the method is applied to the time-dependent relaxation of the (110) surfaces of various III-V and II-VI compound semiconductors, starting from an "ideal" unrelaxed surface.
\end{abstract}

\section{INTRODUCTION}

In a molecular-dynamics simulation ${ }^{1-10}$ one follows the motion of atoms in time. The equations of motion are given by Newton's second law, $\mathbf{F}=m$ a, but are highly coupled-one is faced with a true many-body problem. These equations can be solved numerically, however, and one can follow the motion of a sizable number of atoms in a solid.

In semiconducting (covalently bonded) materials, such simulations promise to yield new information on a number of difficult yet technologically important problems. These include (i) the relaxation and the reconstructed geometries of surfaces and interfaces, and the atomic configurations in semiconducting alloys, (ii) the formation of surface and bulk intrinsic defects, (iii) the kinetics of interface formation, (iv) crystal growth, and (v) vibrational and migration properties of atoms.

A typical molecular-dynamics simulation begins by assuming a simple form for a two-body interaction potential $V\left(r_{i j}\right)$ between atoms $i$ and $j$, such as the Lennard-Jones potential. $^{1-10}$ Such a simple form for the potential gives quite good results for, e.g., the face-centered-cubic noble gas solids. However, in covalently bonded materials, such as $\mathrm{GaAs}$ or $\mathrm{Si}$, the atoms are tetrahedrally bonded. The origin of this bonding is the sharing of electrons between atoms. These electronic-structure-generated interactions are highly directional, and the electronic states are influenced by a large number of atoms, making the forces have a much more complex structure than a simple two-body interaction.

The purpose of this paper is twofold. First, we describe a mathematical formalism by which the many-body forces can be calculated within the simplest type of electronic structure calculation, viz., the tight-binding approximation. The main approximation here is to write the total energy as an electronic part (approximated by the sum of single-electron energy eigenvalues of the Schrödinger equation), and a phenomenological short-ranged two-body central potential. Secondly, we investigate various methods by which these forces may be computed at sur- faces and in the bulk, and apply the method to investigate the relaxation at (110) surfaces of a number of semiconducting compounds.

\section{DETERMINATION OF NONLOCAL FORCES FROM THE ELECTRONIC STRUCTURE}

The tight-binding model is often used to calculate electronic structure properties of semiconductors. In this approximation one uses at least an $s, p_{x}, p_{y}, p_{z}$ basis of atomic orbitals on each atom, which overlap with its neighboring atoms' orbitals to form $s p^{3}$ tetrahedrally directed bonding orbitals of the bulk solid. At a surface or a defect, all of these orbitals may not be bonded to neighboring atoms, and the atom will form variants of the conventional $s p^{3}$ hybrid with a concurrent repositioning of the atoms. To approximate such effects, we write the total energy as a sum of two terms,

$$
U=U^{\mathrm{el}}+U^{R} \text {. }
$$

The electronic term is the energy of the electrons computed within the tight-binding approximation, and will incorporate tetrahedral bonding, bond-bending forces, and rehybridization or dangling bond effects which occur at surfaces or defects. In a bulk crystal this term includes the sharing of electrons amongst atoms and is the attractive force which brings atoms together. The repulsive term we model as a simple short-ranged central potential which keeps the atoms near their bulk equilibrium positions.

Let us now focus on the electronic energy. We compute the electronic energy from the electronic tight-binding Hamiltonian operator

$$
\begin{aligned}
H= & \sum_{\mu, i} \varepsilon_{\mu}(i)\left|\phi_{\mu}\left(\mathbf{r}_{i}\right)\right\rangle\left\langle\phi_{\mu}\left(\mathbf{r}_{i}\right)\right| \\
& +\sum_{\mu, v} \sum_{i, j}^{\prime} V_{\mu \nu}\left(\mathbf{r}_{i}, \mathbf{r}_{j}\right)\left|\phi_{\mu}\left(\mathbf{r}_{i}\right)\right\rangle\left\langle\phi_{\nu}\left(\mathbf{r}_{j}\right)\right|,
\end{aligned}
$$

where $\phi_{\mu}\left(\mathbf{r}_{i}\right)$ is the $\mu$ th atomic orbital $\left(\mu=s, p_{x}, p_{y}, p_{z}\right)$ centered on the atom $i$ located at $\mathbf{r}_{i}$. The quantities $\varepsilon_{\mu}(i)$ are the atomic $s$ and $p$ energies of atom $i$ in the solid, and are 
taken to be independent of the position of the atom. The quantities $V_{\mu v}\left(\mathbf{r}_{i}, \mathbf{r}_{j}\right)$ are the "hopping" matrix elements of the orbital $\mu$ on the atom at $\mathbf{r}_{i}$ with orbital $v$ of atom $j$ at $\mathbf{r}_{j}$, and depend on distance and direction cosines separating atoms $i$ and $j$. There is a large body of literature of such electronic Hamiltonians for electronic structure calculations. ${ }^{11-13}$ Typically the quantities $\varepsilon$ and $V$ are treated as parameters, and are either estimated from first principles or obtained by fitting to more accurate electronic structure calculations.

In this paper we will use the universal nearest-neighbor tight-binding model of Harrison ${ }^{12,14,15}$ which has four hopping matrix elements, $V_{s s \sigma}=\eta_{s s \sigma} \hbar^{2} / m d^{2}$, $V_{s p \sigma}=\eta_{s p \sigma} \hbar^{2} / m d^{2}, \quad V_{p p \sigma}=\eta_{p p \sigma} \hbar^{2} / m d^{2}, \quad$ and $V_{p p \pi}$ $=\eta_{p p \pi} \hbar^{2} / m d^{2}$. Here $d$ is the nearest-neighbor distance and $\eta_{s s \sigma}, \eta_{s p \sigma}, \eta_{p p \sigma}$, and $\eta_{p p \pi}$ are universal constants $-1.32,1.42,2.22$, and -0.63 , respectively, and the $\varepsilon_{\mu}(i)$ [see Eq. (2)] are the $s$ and $p$ atomic energies of the anion and cation taken from Ref. 12. These parameters represent the simplest choice, and no attempt was made to optimize them by fitting to detailed bulk band structures. The advantage of such a "universal" set is they are transferable to less characterized systems and exhibit chemical trends. Harrison's model also prescribes a central repulsive potential between nearest-neighbor atoms of the form $c / d^{4}$, where $c$ is a constant which we fit to reproduce the bulk lattice constant.

The electronic force on atom $i$ is then computed from the derivative of the electronic energy,

$$
\begin{aligned}
\mathbf{F}_{i}^{\mathrm{el}} & =-\frac{\partial U^{\mathrm{el}}}{\partial \mathbf{r}_{i}} \\
& =-2 \sum_{\alpha, o c c}\left\langle\psi_{\alpha}\left|\frac{\partial H}{\partial \mathbf{r}_{i}}\right| \psi_{\alpha}\right\rangle
\end{aligned}
$$

where Eq. (3b) follows from the Hellmann-Feynman theorem, ${ }^{16}$ where the $\psi_{\alpha}$ are eigenstates of $H$, and the sum is over all occupied states. The factor of 2 is from the spin degeneracy.

Equations (3) so far are formal results. In the tightbinding $s-p$ basis, we define a bond order ${ }^{17}$ as

$$
P_{\mu \nu}(i, j)=\sum_{\alpha, o c c}\left\langle\phi_{\mu}\left(\mathbf{r}_{i}\right) \mid \psi_{\alpha}\right\rangle\left\langle\psi_{\alpha} \mid \phi_{v}\left(\mathbf{r}_{j}\right)\right\rangle \text {. }
$$

The bond order is a measure of how much an electron is shared between the orbital $\phi_{\mu}\left(\mathbf{r}_{i}\right)$ of atom $i$ and orbital $\phi_{v}\left(\mathbf{r}_{j}\right)$ of atom $j$. For an ionic crystal, where an electron is transferred from a cation to an anion, $P$ is zero, while for a covalent crystal, where an electron is shared between two directed $s p^{3}$ orbitals,

$$
P_{s p^{3}, s p^{3}}(\text { nearest-neighbor })=\frac{1}{2} .
$$

The electronic force can now be written as

$$
\begin{aligned}
\mathbf{F}_{i}^{\text {el }} & =4 \sum_{j} P_{\mu \nu}(i, j)\left[-\frac{\partial V_{\mu \nu}\left(\mathbf{r}_{i}, \mathbf{r}_{j}\right)}{\partial \mathbf{r}_{i}}\right] \\
& =\sum_{j} \mathbf{F}_{i}^{\text {el }} \text { (due to } j \text { ) }
\end{aligned}
$$

where a sum over repeated indices is understood.
Equation (5) is a very interesting result, since it states that the range of the force is determined entirely by the range of the tight-binding hopping matrix elements. These fall off rapidly with distance (approximately exponentially), and it is a common approximation to keep (in a perfect crystal) only nearest-neighbor hopping interactions. However, the strength of the interaction is proportional to $P$, which is influenced not only by the local environment around atom $i$, but by more distant atoms. Although the model is relatively simple, it includes, in a natural way, the nonlocal $n$-body effects in covalently bonded crystals. We now describe methods to calculate the bond order $P$-an approximate method for the bulk and an exact slab method for surfaces, and we apply the slab method to the relaxation of (110) surfaces.

\section{EVALUATION OF BOND ORDER}

The formal result of Eq. (5) shows that to calculate the force on any given atom $i$ requires knowledge of the bond order $P$, as well as derivatives of the tight-binding interaction parameters $V_{\mu v}\left(\mathbf{r}_{i}, \mathbf{r}_{j}\right) . P$, which represents the sharing of electrons in the formation of the covalent bond, is determined by the electronic structure. In this section we describe two schemes for the calculation of $P$-one for the bulk and the other for the surface of a semiconductor. These are, respectively, the recursion method ${ }^{17}$ and the kspace slab method.

\section{A. Recursion method}

The recursion method ${ }^{17}$ is ideally suited to computing the bond order in a quick, efficient manner-an important consideration in a molecular dynamics simulation, where the electronic structure must be computed repeatedly. The recursion method generates a cluster of atoms, in which the size of the cluster increases with each recursion, ultimately becoming exact for an infinite cluster.

The basic quantities considered are diagonal matrix elements of the Green's function, $G(E)=(E-H)^{-1}$, where $H$ is the tight-binding Hamiltonian. $E$ is the energy, which is taken to mean $E+i \delta$, where $\delta \rightarrow 0^{+}$. The bond order is obtained from matrix elements of the Green's function through

$$
P_{\mu v}(i, j)=-\frac{1}{\pi} \operatorname{Im} \int_{-\infty}^{E_{F}} G_{\mu v}^{i j}(E) d E,
$$

where the $G_{\mu \nu}^{i j}(E)$ are the off-diagonal matrix elements $(i \neq j)$ of the Green's function

$$
G_{\mu v}^{i j}(E)=\left\langle\phi_{\mu}\left(\mathbf{r}_{i}\right)|G(E)| \phi_{v}\left(\mathbf{r}_{j}\right)\right\rangle .
$$

These off-diagonal matrix elements are readily computed from the symmetric and antisymmetric diagonal matrix elements ${ }^{17}$ as $G_{\mu \nu}^{i j}=G_{+}-G_{-}$, where $G_{+}$and $G_{-}$are diagonal matrix elements,

$$
G(E)_{( \pm)}=\frac{1}{2}\left\langle\phi_{\mu}\left(r_{i}\right) \pm \phi_{v}\left(r_{j}\right)|G(E)| \phi_{\mu}\left(r_{i}\right) \pm \phi_{v}\left(r_{j}\right)\right\rangle .
$$

Diagonal matrix elements take the form of a continued fraction 


$$
G_{\alpha \alpha}(E)=\left\langle\phi_{\alpha}|G| \phi_{\alpha}\right\rangle=\frac{1}{E-a_{0}-\frac{b_{1}^{2}}{E-a_{1}-\frac{b_{2}^{2}}{E-a_{2}-\ldots}}}
$$

The coefficients $a_{n}$ and $b_{n}$ can be computed exactly from the Hamiltonian given in Eq. (2). The $a_{n}(n=0,1,2, \ldots)$ are the self-energies of the starting orbital $\phi_{\alpha}(n=0)$ and the orbitals that $\phi_{\alpha}$ couples to, and the $b_{n}$ are the coupling coefficients. Each successive value of $n$ includes a more distant shell of neighbors, and the procedure is exact as $n \rightarrow \infty$.

The simplest approximation is to truncate the continued fraction at $n=1$, viz., set $b_{2}=0$. This approximation includes, in effect, a single orbital of interest and its interactions with its nearest-neighbors, and has the advantage that the theory can be worked out analytically.

To gain insight, let us consider the onsite $(i=j)$ and diagonal $(\mu=v) s, p_{x}, p_{y}$, and $p_{z}$ Green's-function matrix elements for an anion in a tetrahedrally bonded compound semiconductor-i.e.,

$$
G_{s s}^{i i}=\left\langle\phi_{s}\left(r_{i}\right)|G| \phi_{s}\left(r_{i}\right)\right\rangle
$$

and

$$
G_{p_{x} p_{x}}^{i i}=\left\langle\phi_{p_{x}}\left(r_{i}\right)|G| \phi_{p_{x}}\left(r_{i}\right)\right\rangle .
$$

Considering an orbital and its neighbors, the $a$ and $b$ coefficients are

$$
\begin{aligned}
& a_{0}=\varepsilon_{s}(a), \\
& b_{1}=\left(4 V_{s s}^{2}+4 V_{s p_{\sigma}}^{2}\right)^{1 / 2}, \\
& a_{1}=\frac{\varepsilon_{s}(c) V_{s s}^{2}+\varepsilon_{p}(c) V_{s p_{\sigma}}^{2}}{V_{s s}^{2}+V_{s p_{\sigma}}^{2}},
\end{aligned}
$$

for $G_{s s}^{i i}$, and

$$
\begin{aligned}
& a_{0}=\varepsilon_{p}(a), \\
& b_{1}=\left[\frac{4}{3}\left(V_{s p_{\sigma}}^{2}+V_{p p_{\sigma}}^{2}+2 V_{p p_{\pi}}^{2}\right)\right]^{1 / 2}, \\
& a_{1}=\frac{\varepsilon_{s}(c) V_{s p_{\sigma}}^{2}+\left(V_{p p_{\sigma}}^{2}+2 V_{p p_{\pi}}^{2}\right) \varepsilon_{p}(c)}{V_{s p_{\sigma}}^{2}+V_{p p_{\sigma}}^{2}+2 V_{p p_{\pi}}^{2}},
\end{aligned}
$$

for $G_{p p}^{i i}$, where $p p$ is either $p_{x} p_{x}, p_{y} p_{y}$, or $p_{z} p_{z}$. The quantities $\varepsilon_{s}(a), \varepsilon_{s}(c), \varepsilon_{p}(a)$, and $\varepsilon_{p}(c)$ are the atomic $s$ and $p$ energies of the anion and cation [see Eq. (2)]. The onsite diagonal Green's function can be computed analytically in this one recursion approximation to yield an expression in the form

$$
G_{\alpha \alpha}^{i i}(E)=\frac{r_{+}(\alpha)}{E-E_{+}(\alpha)}+\frac{r_{-}(\alpha)}{E-E_{-}(\alpha)}
$$

\begin{tabular}{|c|c|c|c|c|c|c|}
\hline & & lectron ener & $(\mathrm{eV})$ & & sive energy & \\
\hline & $\begin{array}{c}\text { BOM } \\
s p^{3} \\
\end{array}$ & $\begin{array}{l}\text { Recursion } \\
s, p_{x}, p_{y}, p_{z}\end{array}$ & Band structure & $\begin{array}{c}\text { BOM } \\
s p^{3} \\
\end{array}$ & $\begin{array}{l}\text { Recursion } \\
s, p_{x}, p_{y}, p_{z}\end{array}$ & Expt. \\
\hline C & -43.51 & -44.65 & -43.98 & 7.07 & 8.14 & 3.68 \\
\hline $\mathbf{S i}$ & -26.48 & -26.62 & -26.55 & 2.49 & 2.63 & 2.32 \\
\hline $\mathrm{Ge}$ & -26.44 & -26.30 & -26.42 & 2.31 & 2.17 & 1.94 \\
\hline Sn & -22.71 & -22.50 & -22.67 & 1.78 & 1.58 & 1.56 \\
\hline $\mathrm{SiC}$ & -34.33 & -34.94 & -34.56 & 4.55 & 5.16 & 3.17 \\
\hline AlP & -27.32 & -27.62 & -27.40 & 2.43 & 2.73 & 2.13 \\
\hline AlAs & -26.65 & -26.85 & -26.68 & 2.30 & 2.50 & 1.89 \\
\hline $\mathbf{A l S b}$ & -23.73 & -23.84 & -23.78 & 1.84 & 1.94 & \\
\hline GaP & -27.67 & -27.89 & -27.77 & 2.19 & 2.41 & 1.78 \\
\hline GaAs & -27.90 & -27.00 & -26.95 & 2.07 & 2.17 & 1.63 \\
\hline $\mathbf{G a S b}$ & -24.20 & -24.20 & -24.26 & 1.66 & 1.66 & 1.48 \\
\hline InP & -26.23 & -26.45 & -26.30 & 2.10 & 2.32 & 1.74 \\
\hline InAs & -25.65 & -25.77 & -25.67 & 1.97 & 2.09 & 1.55 \\
\hline InSb & -23.05 & -23.09 & -23.10 & 1.55 & 1.59 & 1.40 \\
\hline $\mathrm{ZnSe}$ & -28.66 & -29.11 & -28.74 & 1.46 & 1.91 & 1.29 \\
\hline $\mathrm{ZnTe}$ & -25.23 & -25.58 & -25.33 & 0.99 & 1.34 & 1.14 \\
\hline
\end{tabular}

Here we interpret $E_{+}(\alpha)\left[E_{-}(\alpha)\right]$ as the bonding (antibonding) energy level associated with the $\alpha$ orbital. The residues are $r_{+}$and $r_{-}$.

Let us consider the question of whether such a small number of recursions is a useful approximation to the

TABLE I. Comparison of different calculations of the one-electron energies. The first three columns show the one-electron energies computed using the bond orbital model (BOM), the recursion method [Eq. (13)], and the full band-structure calculation. The last three columns compare the cohesive energies determined within a BOM framework using the BOM and recursion one-electron energies. The experimental values are from tabulations in Ref. 12. 
electronic energy. An approximate form of the oneelectron energy can be obtained from the expressions above:

$$
\begin{aligned}
u(\text { electronic })=\frac{1}{4}[ & E_{+}\left(s_{a}\right)+E_{+}\left(s_{c}\right) \\
& \left.+3 E_{+}\left(p_{x a}\right)+3 E_{+}\left(p_{x c}\right)\right],
\end{aligned}
$$

where $u$ (electronic) is the single-particle energy per bond (two electrons). Here $E_{+}$is the bonding energy in Eq. (12) and is obtained by solving a quadratic equation involving the $a$ and $b$ coefficients. This form is very similar to the bond orbital model (BOM) developed by Harrison and coworkers ${ }^{12,14,15}$ in terms of $s p^{3}$-hybrid orbitals. The difference is that the recursion method can easily be extended to include more distant neighbors, and the electronic structure is free to take on any form of hybridization. In Table I we compare the accuracy of the simple analytical expression of Eq. (13), the BOM, and the exact band-structure calculation

$$
\frac{v_{c}}{4(2 \pi)^{3}} \sum_{n, o c c} \int d^{3} k E_{n}(\mathbf{k}),
$$

all using the same Hamiltonian Eq. (2). Also shown is the cohesive energy obtained from both the recursion method and the BOM. The cohesive energy is computed following Harrison and co-workers ${ }^{12,14,15}$ where we use a central repulsive potential between nearest neighbors,

$$
V^{R}=\frac{\eta V_{2}^{2}}{\left|\left\langle\varepsilon_{h}\right\rangle\right|}
$$

where $\left\langle\varepsilon_{h}\right\rangle$ is the average hybrid energy of the anion and cation,

$$
V_{2}=\frac{-3.22 \hbar^{2}}{m d^{2}}
$$

and $\eta$ is a constant adjusted to reproduce the nearestneighbor distance $d$.

A comparison of the one-electron energy computed using just one recursion with the exact band-structure calculation shows reasonably good agreement. Thus, for those atoms in a molecular-dynamics simulation which are nearly tetrahedrally bonded, the recursion method will give reasonably accurate one-electron energies with just one recursion. The recursion method, of course, can be

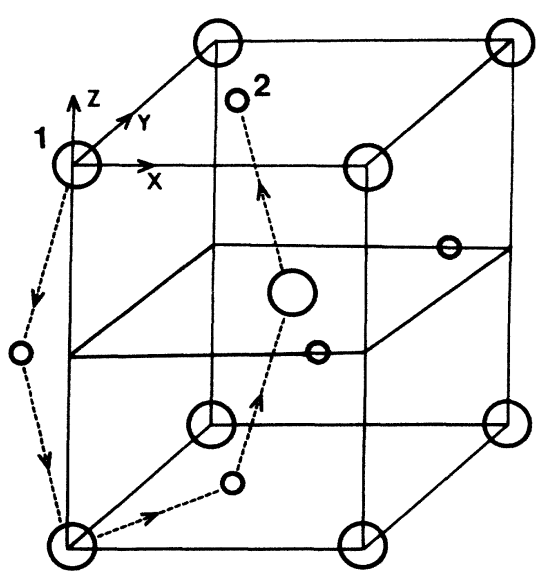

FIG. 1. Atomic geometry near an unrelaxed (110) surface of GaAs or other compound semiconductor with zinc-blende structure. The large and small circles represent the two species of atoms. There is no direct interaction between an $s$ orbital of surface atom 1 and a $p_{z}$ orbital of surface atom 2. Instead, there is only an indirect interaction via rings of the kind indicated by the dashed lines with arrows. This implies, as discussed in the text, that a large number of recursions are required to treat surface relaxation in the recursion method.

made more precise by employing more recursions-i.e., by including more distant atoms.

\section{B. Slab method for surfaces}

In contrast to the bulk, we have found that the recursion method converges slowly for atoms at a surface. The reason for this is illustrated in Fig. 1, where we show the top three layers of an ideal unrelaxed (110) surface of a compound semiconductor such as GaAs. Since the bonding at the surface is not tetrahedral (one bond is broken), a force exists on these atoms, so that the surface atoms relax to new positions. We now consider their motion in the $z$ direction (perpendicular to the slab).

Consider the force in the $z$ direction of surface atom labeled 1 due to surface atom 2. From Eq. (5), this force is given by

$$
\begin{aligned}
{\left[F_{1}(\text { due to } 2)\right]_{z} } & =-4 \sum P_{\mu v}(12) \frac{\partial V_{\mu v}}{\partial z_{1}}(12) \\
& =-4\left[P_{s z}(12) \frac{\partial V_{s z}}{\partial z_{1}}(12)+P_{z s}(12) \frac{\partial V_{z \sigma}}{\partial z_{1}}(12)+P_{z \sigma}(12) \frac{\partial V_{z \sigma}}{\partial z_{1}}(12)+P_{\sigma z}(12) \frac{\partial V_{\sigma z}}{\partial z_{1}}(12)\right),
\end{aligned}
$$

where $\sigma$ refers to a $p$ orbital polarized along the axis of the bond between the two surface atoms. The central repulsive force in the $z$ direction is identically zero, so the net force is entirely electronic. The last expression in Eq. (15) follows from the symmetry of the unrelaxed surface and the nearest-neighbor tight-binding Hamiltonian. The first two terms in the last expression of Eq. (15) involve the coupling of the $s$ and $p_{\pi}$ orbitals of the two surface atoms, while the last two terms involve coupling a $p_{\sigma}$ orbital with a $p_{\pi}$ orbital. Note, however, that the direct $s-p_{\pi}$ and $p_{\sigma}-p_{\pi}$ interactions vanish, leaving only indirect interactions through electron hopping via more distant atoms. (See Fig. 1. An example of an indirect interaction is indicated by the dashed lines with arrows.) In the bulk 
of a perfect crystal, even these indirect interactions vanish. Only at a surface or other region of reduced symmetry are these indirect interactions nonzero. At the surface, the electron must circulate at least around six-membered rings (see Fig. 1) in order for this indirect interaction not to vanish. Thus large clusters must be considered, and the important physics is not given by a few atoms-i.e., a few recursions. We find this indirect interaction to be the dominant force on a surface As atom and that it acts perpendicular (noncentral) to the bonding direction between the two surface atoms. From the calculations of Sec. IV, we find this force in GaAs (atom 1 is As) to be +0.71 $\mathrm{eV} / \AA$, while the recursion method for $n=2$ yields +0.03 $\mathrm{eV} / \AA$ and for $n=4$ yields $+0.33 \mathrm{eV} / \AA$. Similar indirect interactions have been previously noted between adatoms. $^{18}$

To include these multiatom effects at a surface, we have to consider a finite slab exactly. In this technique we use a large unit cell and impose periodic boundary conditions so that each unit cell is identical, allowing $\bar{k}$-space ( $\bar{k}$ parallel to slab) techniques to be used. The bond order is then computed exactly by diagonalizing, the $\bar{k}$ dependent Hamiltonian matrix. This technique can be used for any size unit cell we choose, but has the disadvantage that large matrices must be diagonalized. This technique is easy to use and formulate and is an ideal method for the application of (110) surface relaxation of compound semiconductors, where only a small unit cell is required.

\section{APPLICATION TO SEMICONDUCTOR (110) SURFACE RELAXATION}

Experimentally it is known that the atoms at a (110) surface occupy sites different from those expected by simply terminating a bulk solid. ${ }^{19,20}$ For a III-V compound such as GaAs, the anion (As) moves out of the surface plane toward vacuum, and the cation $(\mathrm{Ga})$ moves downward toward the bulk. The physical origin of this effect is that the cation with three electrons seeks a planar geometry of $s p^{2}$ orbitals, and the anion with five electrons seeks a pyramidal configuration with three $p$ electrons bonding at right angles and two electrons in $s$ states.

Chadi's ${ }^{21}$ pioneering work on this problem has shown that this surface relaxation may be calculated in a tightbinding model. As a test example, we have reexamined this problem using our method which computes the force from the electronic structure using the HellmannFeynman theorem and the repulsive potential of Eq. (14), and solves the many-body equations of motion using molecular dynamics.

The molecular dynamics simulation was performed on a slab of six layers whose normal is the [110] direction. The six layers comprised a large unit cell and each layer of the unit cell contained one anion and one cation. We used rigid periodic boundary conditions so that all the atoms in the next unit cell were constrained to move exactly as those in the central large unit cell. Since the reconstruction is $(1 \times 1)$, this is a reasonable procedure. The bond order $P$ was computed using one special twodimensional $k$ point, ${ }^{22} k_{s p}=(1 / 2 a, 1 / 2 b) \pi$. Here $a$ and $b$ are the dimensions of the rectangular two-dimensional primitive translation vectors, where $a$ is the length of the cube edge of the underlying face-centered-cubic lattice and $b=a / \sqrt{2}$.

Denoting the time by $t$, we start the atoms at $t=0$, with zero velocity and at the positions they would have in a terminated bulk crystal. The force on a surface As atom (in GaAs) is nearly perpendicular to the slab and away from the slab. The force on a surface $\mathrm{Ga}$ atom is mainly into the slab, but has a non-negligible component toward the line of As surface atoms. Every six steps we quench the system. That is, all the kinetic energy is removed, and the atoms are set back to zero velocity. In Fig. 2 we give a measure of how fast a six-layer GaAs slab reaches its equilibrium position. The ordinate is the "temperature" which is computed from the average kinetic energy, and is not the true thermodynamic temperature. It does, however, give a measure of the remaining forces on the atoms and how fast they are moving. The solid line is a guide to the eye, and was drawn only through the points just before a quench. An exception to this is that every time step is shown for the initial six time steps before the first quench, which give the peak behavior. We see that the temperature rises rapidly (to about $1000 \mathrm{~K}$ ) during the first six time steps, due to the large initial forces, but decreases rapidly thereafter as the energy is removed due to quenching and the equilibrium positions are attained.

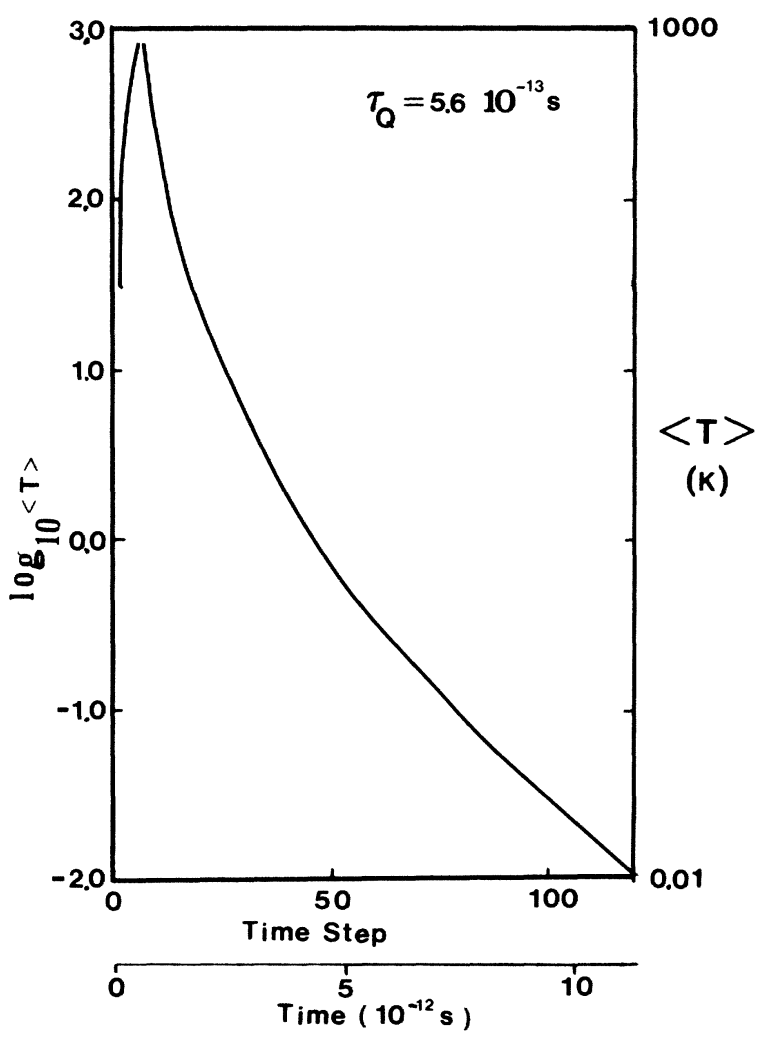

FIG. 2. "Temperature" $T_{K E}$, defined by $\frac{3}{2} k_{B} T_{K E}=$ average kinetic energy of slab, as a function of time. The system is quenched after every sixth time step; i.e., all velocities are set equal to zero when $t=\tau_{Q}$, where $t$ is the time. 
A common measure of the (110) surface lattice relaxation is the relaxation angle $\theta .^{21}$ Choosing the unrelaxed surface anion to be the origin, and the unrelaxed surface cation at $(a / 4, b / 2,0)$ where the $z$ direction is normal to the slab, the relaxation angle is given $b y^{21}$

$$
\tan \theta=\left|\left(z_{c}-z_{a}\right) /\left(x_{c}-x_{a}\right)\right| .
$$

Experimentally, ${ }^{19,20} \theta \approx 27^{\circ}$ for GaAs. In Fig. 3 we show $\theta(t)$ for a number of different semiconducting III-V and II-VI compounds and SiC. Again we quench every sixth time step to damp out the oscillations. We find, in agreement with the subset of compounds considered by Chadi, that the surface relaxation angle is nearly a constant for all (110) surfaces. We find $\theta$ to vary only from about 25 to 30 degrees for twelve different compounds. Notice that InP approaches its final value of $\theta=27.4^{\circ}$ more slowly than the rest. This is due to the large mass difference between In and P. The In atom responds only slowly to its force.

Recently Duke et al. ${ }^{23}$ have also considered this problem and have determined relaxation angles of $29.5^{\circ}, 28.7^{\circ}$, $29.6^{\circ}, 31.6^{\circ}, 32.2^{\circ}$, and $30.4^{\circ}$ for GaAs, GaP, GaSb, InP, InAs, and InSb, respectively. For the III-V materials, our values of $\theta$ are consistently higher than Chadi's and lower than Duke's. All three calculations are similar in spirit. Ours is perhaps the simplest since we use Harrison's universal model, so that detailed fits to bands are not required and there is only one undetermined constant $\eta$ in Eq. (14).

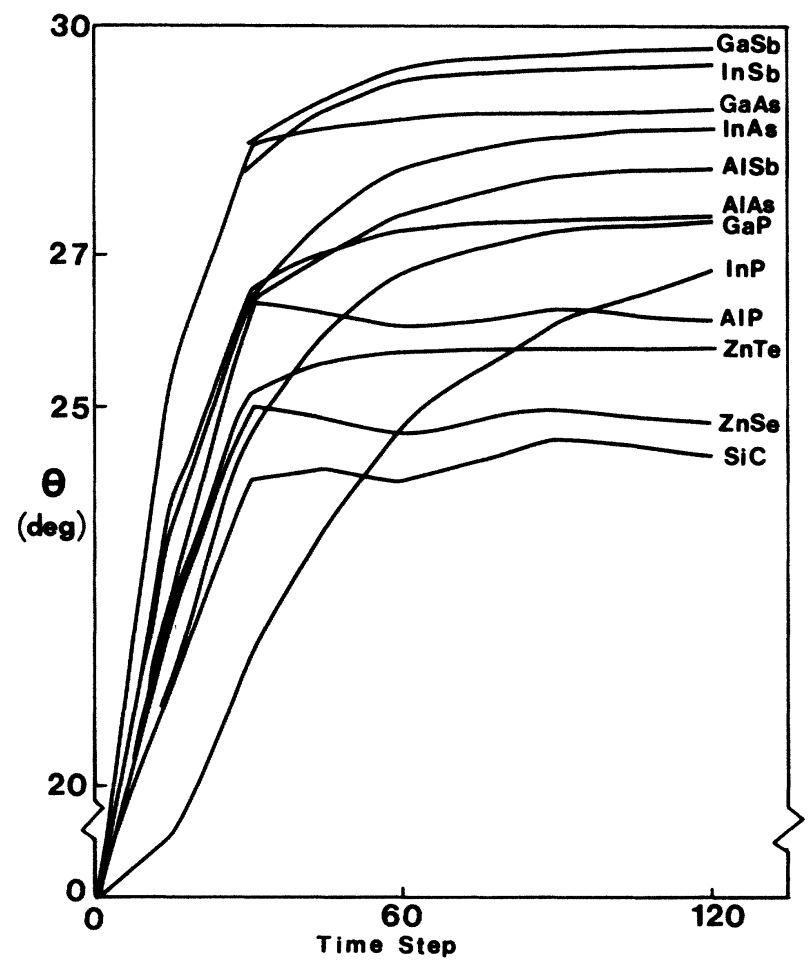

FIG. 3. Relaxation angle $\theta$, given by Eq. (16), as a function of time for the 12 semiconductors shown. The atoms initially have the positions of an "ideal" unrelaxed surface; quenching again occurs every sixth time step, with different time steps chosen for the different materials.
Finally, in Table II we tabulate the final lattice positions of the top three layers using the coordinate system of Fig. 1. In the table we have defined a new zero for the $x$ coordinate since a large shift of the surface atoms also moves subsurface atoms to conserve the center of mass. For those compounds treated by Chadi using an energy minimization technique, the two methods are in substantial agreement.

The atomic displacements perpendicular to the surface can be measured more accurately than the parallel displacements. A common measure of the perpendicular displacements is $\Delta_{1},{ }^{24}$ which in our notation is $\delta z_{a}-\delta z_{c}$, listed in Table II. The values of $\Delta_{\perp}$ show nearly a factor of 2 difference in the compounds studied, ranging from $\sim 0.4-0.8 \AA$. Physically the trends can be understood by noting the tendency of the cation to become planar with its neighbors. In the III-V materials, a surface cation is surrounded by only three neighbors and the energy is reduced by forming an $s p^{2}$ bonding configuration. Assuming for the moment that the bond lengths are preserved and only a rigid rotation occurs, the condition that the bonding becomes planar is $\Delta_{1}=0.33 d$, where $d$ is the bond length. We thus write

$$
\Delta_{\perp}=0.33 d+C,
$$

where $C$ indicates correction terms which allow the atoms to deviate from a precisely planar configuration, and to have nonconstant bond lengths. An inspection of the calculated values of $\Delta_{\perp}$ listed in Table II reveals that the IIIV's and SiC do show such a trend. These materials can be least-squares fitted to

$$
\Delta_{\perp}=0.33 d-0.159+0.051(d-2.47) .
$$

Here $d$ and $\Delta_{1}$ are in $\AA$, and the average bond length of these materials is $2.47 \AA$. The average correction $C$ in Eq. (16) is $-0.159 \AA$, and itself has a small bond-lengthdependent correction given by the last term of Eq. (18). The range of $C$ values is $-0.20 \AA$ for $A 1 P$ to $-0.11 \AA$ for GaSb. The IV-IV material SiC also fits in this scheme with a value of $C$ of $-0.18 \mathrm{~A}$. This is due to the charge exchange from $\mathrm{Si}$ to $\mathrm{C}$, making it behave in this respect as a III-V. Noticeably different are the II-VI materials, with $C$ values of -0.25 and $-0.24 \AA$ for $\mathrm{ZnSe}$ and $\mathrm{ZnTe}$, respectively. The $\mathrm{Zn}$ atom with only two electrons is not driven to form a planar configuration. Duke ${ }^{23}$ has established empirical trends in the data of a number of III-V and II-VI compounds and finds

$$
\Delta_{\perp}=0.33 d-0.156+0.146(d-2.47) .
$$

This is in substantial agreement with our least-squares fit of our calculated values given in Eq. (18). However, the data seem to indicate a larger variation with lattice constant. The slope in Duke's fit is 0.48 , while our calculated result is 0.38 . More importantly, the II-VI materials appear to fit Eq. (19) as well as the III-V's do. Our tightbinding results show the II-VI's to differ substantially. This may be an indication of ionic or charge transfer effects not included in the tight-binding Hamiltonian which may be more important for these materials. 


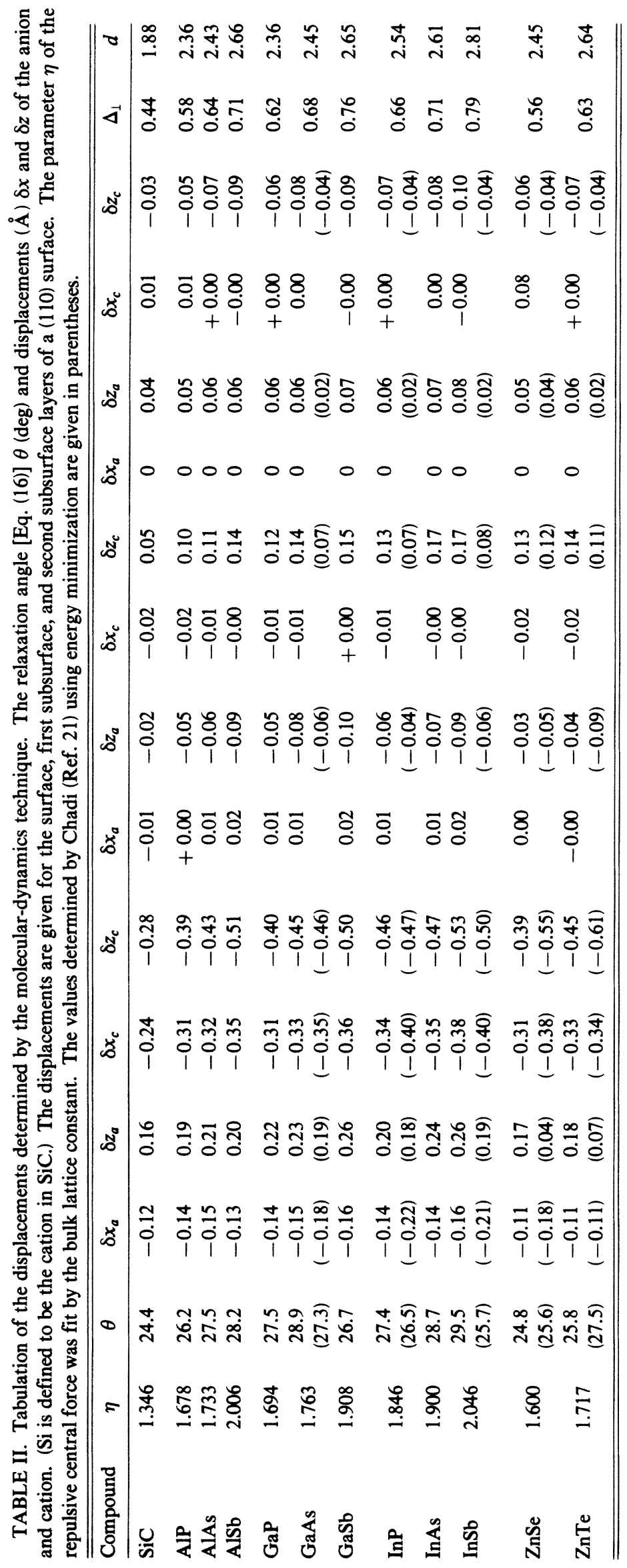




\section{SUMMARY}

We have developed a scheme for performing molecular-dynamics calculations in semiconductors using the electronic structure in the tight-binding approximation to generate the forces. The method has the advantage that the range of the force depends only on the range of the tight-binding interactions, but that the strength of the force involves the electronic structure which is sensitive to the arrangement of atoms outside the range. The scheme thus includes naturally the noncentral $n$-body interac- tions. As an example, we have considered relaxation at semiconductor (110) surfaces and found agreement with earlier calculations and experiment.

\section{ACKNOWLEDGMENTS}

We thank the U. S. Office of Naval Research (Arlington, VA) for their support, which made this work possible (N00014-85-K-0442) and (N00014-82-K-0447). Additional support was provided by the Robert $A$. Welch Foundation.
${ }^{1}$ B. M. Alder and T. W. Wainwright, J. Chem. Phys. 31, 459 (1959).

${ }^{2}$ A. Rahman, Phys. Rev. 136, A405 (1964).

${ }^{3}$ R. E. Allen, F. W. de Wette, and A. Rahman, Phys. Rev. 179, 887 (1969).

${ }^{4}$ F. H. Stillinger and A. Rahman, J. Chem. Phys. 60, 1545 (1974).

${ }^{5}$ F. F. Abraham, J. Vac. Sci. Technol. 2, 534 (1984), and references therein.

${ }^{6}$ H. C. Andersen, J. Chem. Phys. 72, 2384 (1980).

${ }^{7}$ M. Parrinello and A. Rahman, in Melting, Localization, and Chaos, edited by R. K. Kalia and P. Vashista (North-Holland, New York, 1982).

${ }^{8}$ I. K. Schuller and A. Rahman, Phys. Rev. Lett. 50, 1377 (1983).

9J. C. Tully, Surf. Sci. 125, 282 (1983).

${ }^{10}$ R. N. Barnett, C. L. Cleveland, and U. Landman, Phys. Rev. Lett. 54, 1679 (1985).

${ }^{11}$ D. J. Chadi and M. L. Cohen, Phys. Status Solidi B 68, 405 (1975).

12W. A. Harrison, Electronic Structure and the Properties of Solids (Freeman, San Francisco, 1980), and references therein.
${ }^{13}$ P. Vogl, H. P. Hjalmarson, and J. D. Dow, J. Phys. Chem. Solids 44, 365 (1983).

${ }^{14}$ E. A. Kraut and W. A. Harrison, J. Vac. Sci. Technol. B 2, 409 (1984).

${ }^{15}$ R. Enderlein and W. A. Harrison, Phys. Rev. B 30, 1867 (1984).

${ }^{16}$ R. P. Feynman, Phys. Rev. 56, 340 (1939).

${ }^{17} \mathrm{~V}$. Heine, in Solid State Physics, edited by H. Ehrenreich, F. Seitz, and D. Turnbull (Academic, New York, 1980) Vol. 35.

${ }^{18}$ T. L. Einstein and J. R. Schrieffer, Phys. Rev. B 7, 3629 (1973).

${ }^{19}$ S. Y. Tong, A. R. Lubinsky, B. J. Mrstik, and M. A. van Hove, Phys. Rev. B 17, 3303 (1978)

${ }^{20}$ A. Kahn, E. So, P. Mark, and C. B. Duke, J. Vac. Sci. Technol. 15, 580 (1978); C. B. Duke, R. J. Meyer, and P. Mark, ibid. 17, 971 (1980).

${ }^{21}$ D. J. Chadi, J. Vac. Sci. Technol. 15, 631 (1978); 15, 1244 (1978); Phys. Rev. B 18, 1800 (1978).

22S. L. Cunningham, Phys. Rev. B 10, 4988 (1974).

${ }^{23}$ C. B. Duke, C. Mailhiot, A. Paton, D. J. Chadi, and A. Kahn, J. Vac. Sci. Technol. B 3, 1087 (1985).

${ }^{24}$ C. B. Duke, J. Vac. Sci. Technol. B 1, 732 (1983). 